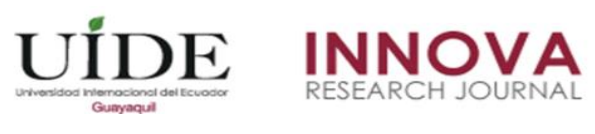

INNOVA Research Journal, ISSN 2477-9024

(Mayo, 2017). Vol. 2, No.5 pp. 14-27

DOI: https://doi.org/10.33890/innova.v2.n5.2017.162

URL: http://revistas.uide.edu.ec/index.php/innova/index

Correo: innova@uide.edu.ec

\title{
Origen de la economía ecológica y su aplicación en las empresas Ecuatorianas
}

\section{Origin of the ecological economy and its application in Ecuadorian companies}

Stephanie Marcela Delgado Estrada

Maritza Mariela Peñafiel

Universidad de Guayaquil, Ecuador

Autor por Correspondencia: stephi_delgado@hotmail.com, maritza.penafiele@ug.edu.ec

Fecha de recepción: 3 de Febrero de 2017 - Fecha de aceptación: 28 de Abril de 2017

Resumen: El cambio climático de la última década ha generado que en el planeta en ciertas zonas exista un aumento de la temperatura, exista un deshilo de los polos y además un aumento de las lluvias, huracanes, terremotos entre otros. Se han llevado a cabo cumbres promovidas por el sector privado y a nivel de naciones con la finalidad de firmar acuerdos, impulsar créditos sobre proyectos que cuiden el medio ambiente, pero los intereses de generar beneficios sin importar el impacto al ecosistema han sido mayores. Producto del bienestar del planeta se ha fomentado aplicar una economía ecológica en donde pone como punto de partida emplear materias primas recicladas para iniciar el proceso de producción, sin embargo esto requiere altos costos que impactarían al precio final, convirtiendo menos competitivos a las empresas que lo aplican. En el Ecuador el Gobierno Nacional ha realizado esfuerzos por disminuir los efectos de gas invernadero aplicando los llamados impuestos verdes en el transporte e incentivando el cambio de automotores a través de planes de renovación, el trabajo se concluyó que la concientización de la contaminación proviene de la iniciativa de todos los actores del mercado, consumidores finales, clientes, empresas, gobierno; si los mismos proveen de actividades que vayan a favor del planeta, y pagan un precio justo por procesos más limpios con productos a base de materiales reciclados, en gran parte cambiarían ese enfoque de economía que actualmente sigue en marcha donde el consumo es considerado un desarrollo en una nación.

Palabras claves: economía ecológica; contaminación; consumo; créditos; cumbres climáticas

Abstract: The climate change of the last decade has generated a rise in temperature in the planet, there is a collapse of the poles and an increase in rainfall, hurricanes, earthquakes and others. Summits promoted by the private sector and at the level of nations have been carried out with the purpose of signing agreements, promoting credits for projects that take care of the environment, but the interests of generating benefits, regardless of the impact to the ecosystem, have been greater. A product of the welfare of the planet has been encouraged to apply a green economy where it uses as a starting point to use recycled raw materials to start the production process, however this requires high costs that would impact the final price, making less competitive companies Apply. In Ecuador, the National Government has made efforts to reduce the effects of greenhouse gas by applying the so-called green taxes on transportation and encouraging the change of automobiles through renovation plans, the work concluded that the awareness of pollution comes from the Initiative of all market players, end consumers, customers, companies, government; If they provide activities that go in favor of the planet and pay a fair price for cleaner processes with products based on recycled materials, would largely change that approach to economy that is currently underway where consumption is considered a development In a nation.

Key Words: ecological economics; pollution; consumption; credits; climatic summits 


\section{Introducción}

Dentro de los factores administrativos que se recomienda para el manejo de las empresas se encuentra el manejo óptimo de los recursos, es decir emplear medios de producción con materia prima mínima a fin de elaborar un mayor número de bienes u ofertar servicios sin medir el impacto ecológico en el medio ambiente, ya que se trata de presupuestar los recursos y con ello obtener el máximo de utilidad posible.

Un ejemplo de impacto ecológico es el uso del papel en todo el proceso administrativo de una empresa, ya que se requiere mostrar informes, anotar aspectos de control como conteo físico de inventarios, registrar la entrega de mercadería, o hasta tomar los datos de los clientes para luego enviar proformas u ofertar de lo que demandan; es decir en todo se usa el papel.

Para obtener el recurso del papel se utiliza como materia prima los árboles, sin embargo estos son necesarios para la generación de oxígeno en el planeta, ya que ellos transforman el dióxido de carbono que expulsan todos los seres vivos y las maquinarias que nacieron a través de la revolución industrial.

Pero el uso de estudios ecológicos que determinen el impacto de las actividades de producción y comercialización de una empresa demandan recursos que no son factores dependientes de la generación de ingresos por lo tanto las empresas no tienen la necesidad de hacerlos y son pocos los países que obligan a las compañías a tener en consideración el medio ambiente para desarrollar sus modelos de negocios.

El concepto de economía ecológica nace por la importancia de concientizar a las organizaciones sobre el impacto en el medio ambiente, naturaleza y efectos climáticos, que generarían mayores gastos en el modo de comercialización, probablemente disminuyan el beneficio en la venta, pero sería un ejemplo para los competidores de lograr sostenibilidad en las actividades que desarrollar y a la vez cuidando que sus actividades causen el menor impacto posible al planeta.

En el presente artículo de investigación se muestra a fondo el concepto de economía ecológica, de dónde nació esta idea y cómo actualmente los actores de la economía han procedido para que todas las empresas agreguen en su modelo de negocios actividades adicionales que impacten en menor proporción al medio ambiente.

\section{Estudio del arte.}

Las empresas según la actividad de la cual ofertan sus productos o servicios puede ser industriales, comercialización si se trata de un producto tangible, si el producto es intangible se clasifica en servicios, cada una de ellas tiene la particularidad de trabajar en función a su presupuesto, ya que incurren costos y gastos para la generación de dichos ingresos, algunos analistas financieros emplean la herramienta del punto de equilibrio para conocer cuánto nivel de ventas deben incurrir para poder cubrir dichos rubros. 
El problema radica cuando existe una gestión en la labor de la gerencia al compararse el rendimiento de la compañía con otra de iguales características, ya que podría darse el caso que la misma incurra en valores inferiores y haya generado un beneficio mayor en el modelo de negocios. Aquí es donde se comienza a catalogar al administrador como un derrochador de recursos o con la falta de conocimientos para lograr manejar los recursos de manera eficiente.

Para Fred (2013) indica que la toma de decisiones debe ser basada en el manejo eficiente de los recursos, es decir cumplir con una meta u objetivo mínimo pero con la característica de lograrlo en el menor tiempo posible y empleando el menor número de recursos para cumplirlo. Es aquí donde el concepto de economía a escala que se basa en alargar los costos para bajar los unitarios al máximo mientras mayor es el número de unidades producidas.

Por lo tanto la administración en una empresa siempre busca alcanzar un objetivo independientemente si las técnicas usadas afectan o no al ecosistema, modifican la naturaleza donde radica la fábrica o están las oficinas de la compañía. Es decir se forma un conflicto entre la sociedad que busca cada vez mejorar su calidad de vida demandando productos que le crean el mayor beneficio y la naturaleza que está siendo afectada por el empleo de dichos recursos con residuos que no son procesados de manera correcta y que a su vez crean serios daños al ecosistema.

En la actualidad la sociedad valoriza mucho el dinero como la fuente para obtener o realizar una acción pero que a su vez no contempla un cuidado del plantea, muchos grupos en protección han surgido porque necesitan que se controle la actividad industrial que daña al ecosistema y que afecta a todos los habitantes.

A continuación se muestra algunos conceptos que han permitido el surgimiento de la economía ecológica como una ciencia que busca resolver los conflictos entre la sociedad y la naturaleza, de manera que los cumplan sus objetivos y que estos a su vez puedan dar pautas para que las empresas sigan el ejemplo, abasteciendo al mercado u ofertar servicios pero sin dañar los ecosistemas.

\section{Concepto de ecología.}

En referencia a Cely (2011) es ante todo una ciencia de síntesis, que trata de las relaciones de los organismos con su medio ambiente. El estudio de estas relaciones ha llevado al hombre a entender la complejidad del mundo viviente, tratando de explicar cómo ésta complejidad se estructura y funciona en diferentes niveles de jerarquía y organización. Como se entrelazan materia - energía - organización son aspectos claves para el entendimiento de los ecosistemas.

Los más importante de la ecología es que haya llegado a demostrar claramente las interrelaciones que existen entre los seres vivos y sus ambientes con un enfoque globalizador.

Concepto de economía.

Por economía se entiende como el conjunto de instituciones y procedimientos para cubrir de un modo ordenado, duradero y seguro las necesidades humanas de bienes y servicios escasos, 
que posibilitan al individuo y a las unidades sociales el desarrollo exigido por la naturaleza del hombre, en cuanto ser individual y social (Gómez, 2012).

Como lo ha señalado el autor, la definición de economía parte como un fin o tiene un objetivo, el desarrollo y perfeccionamiento de todas las posibilidades, exigencias y aptitudes que, por creación, están ínsitas en la naturaleza del hombre, en segundo lugar, hace notar que tal fin no puede alcanzarse sino en el marco de la serie de instituciones jurídicas y sociales estables.

Relación entre la economía y la ecología.

De acuerdo a los conceptos expuestos por economía y ecología sus finalidades son totalmente opuestas, ya que la primera identifica que el ser humano demanda necesidades y que dichas acciones deben ser cubiertas por instituciones u otras personas para generar un desarrollo enfocado en la entrega de objetos materiales a cambio de un bien económico, mientras que la segunda trata de estudiar la relación entre el ser humano y la naturaleza sin que ninguno ponga condicionamientos para garantizar dicha relación.

A continuación se muestra un esquema que describa de manera más didáctica las finalidades de cada uno de estos conceptos:

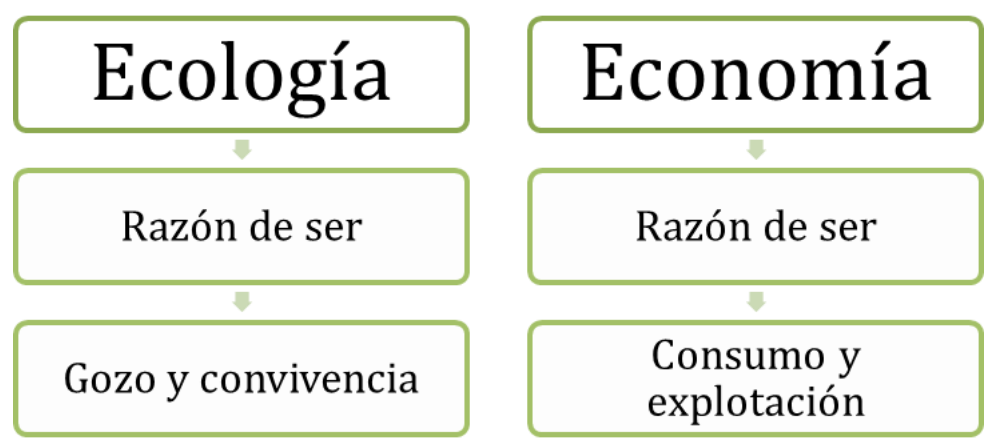

Figura 1. Relación entre la ecología y la economía.

En la figura 1 se muestra la relación que existe entre la ecología y la economía, evidenciando que son dos ciencias distintas que persiguen objetivos diferentes, sin embargo algunos analistas la confunden diciendo que la economía es una forma que las personas puedan convivir en el medio, ya que tienen los recursos materiales para proveerse de los recursos básicos que son alimentación, salud y educación pero no estudian que estos objetivos se los obtiene solamente con el empleo de recursos que no son renovables y que producen un daño al medio ambiente.

Un ejemplo de cómo la economía relaciona este concepto con ecología de manera perjudicial es el transporte, siendo el medio que ha existido desde la antigüedad y que gracias a la revolución industrial ha empleado combustibles no renovables que exponen al ambiente gases de efecto invernadero que calientan el planeta. Aquí se ha cubierto la necesidad del ser humano de traslado sin embargo para el planeta ha generado que cambie su clima, exponga humo y a su vez contribuya a generar enfermedades a los mismos seres humanos. 


\section{Concepto de economía ecológica.}

Según Fernández (2013) la economía ecológica es la ciencia de la gestión de la sostenibilidad, entendiendo por sostenibilidad la viabilidad en el tiempo de un sistema el cual está condicionado por sus intercambios con el entorno físico. Esta ciencia trata de encontrar soluciones teóricas que le permitan integrar en los modelos tradicionales, las consecuencias o los efectos externos no deseados de la actividad económica, es decir, en la internalización, a través de los precios, de las externalidades ambientales negativas.

Algunos economistas llaman externalización de costes tanto a la falta de incorporación de los mismos a la contabilidad empresarial, como también a la ausencia de estos cargos en el precio final de un producto o servicio que está dispuesto a pagar el consumidor.

En esta ciencia tiene un punto débil es que en muchas ocasiones es imposible adjudicar valores monetarios a las externalidades, porque en su mayoría son inciertas, desconocidas o irreversibles, por lo que estamos ante un concepto económico muy politizado, ya que, en teoría, la sociedad en su conjunto tiene que fijar cuáles son los límites ecológicos de la economía. Y si no se hace de una forma global que puede generar pérdidas de competitividad nacionales.

Tanto la economía ecológica como la economía tradicional afirman ocuparse de la gestión de lo útil y lo escaso, pero interpretan estos términos de manera distinta, tal como se explicó en la figura 1 donde estas dos ciencias, la economía y la ecología tienen objetivos distintos.

Para Barcia (2012) la economía tradicional solo se ocupa de aquello que, siendo de utilidad directa para los seres humanos resulta además apropiable, valorable y producible. Es decir que es un flujo de información circular cerrado del dinero entre la producción y el consumo, auto sostenido, que ignora totalmente los aspectos ambientales de la actividad económica.

La economía en materia política en referencia Emery (2011) indica que el desarrollo de una nación está representada por la mayor cantidad de bienes de consumo que se elaboren, ya que la presencia de industrias demandan plazas de trabajo que a su vez emplean a otros individuos, de manera que todos reciban un beneficio económico para intercambiarlo por lo menos de servicios básicos y alimentación.

Esta economía tiene efectos negativos, un ejemplo de ello es China, país industrializado que está superando a otras economías por su aumento de niveles de producción y la competitividad en cualquier producto. Sin embargo sus efectos negativos en la ecología, han hecho que sus habitantes sufran las consecuencias de impulsar un gran nivel de producción sin precautelar un adecuado procesamiento de la basura o de los residuos de las fábricas. 


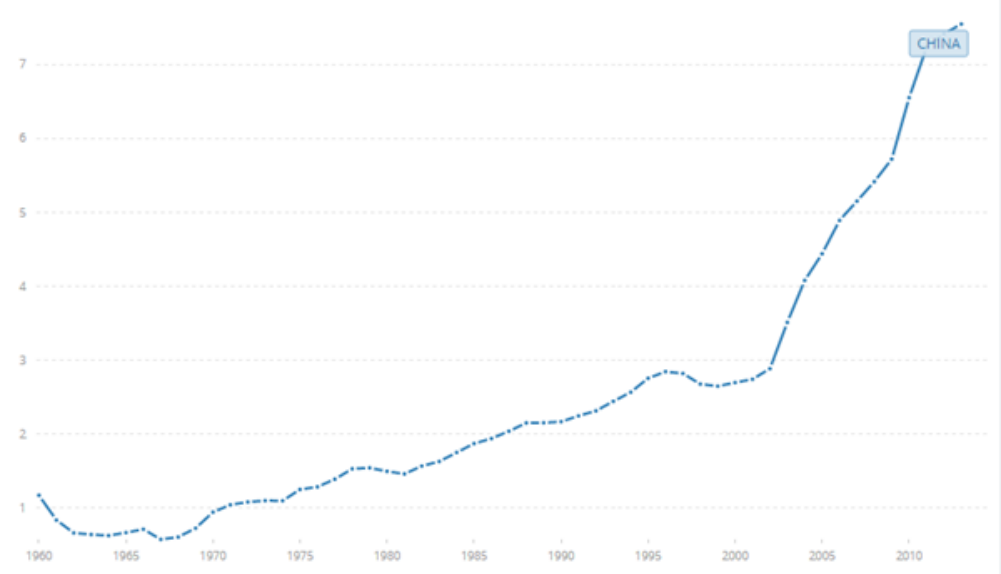

Figura 2. Emisiones de CO2 de China en toneladas métricas per capital 1960 - 2013

Según el Banco Mundial (2016) a finales de los años 90 las ciudades que se cerca de las dos Coreas y Japón han sufrido grandes problemas de emisiones de $\mathrm{CO} 2$ debido al aumento de energía que demanda la industria y el transporte abastecida de combustibles fósiles, sumado a esto problemas de erosión en casi un tercio del territorio chino. Frente a este problema se tiene que China no hace el mayor esfuerzo para corregir este problema ya que no representa beneficio económico frenar a las empresas que emplean a millones de chinos de manera anual.

La economía ecológica considera que toda la biosfera y los recursos pueden ser a la vez escasos y útiles, con independencia de que sean o no valorados en el mercado. Considera la economía como un subsistema abierto dentro de la biosfera que considera los factores físicos de la actividad económica como la necesidad de:

- Un suministro adecuado de energía y materias primas, que depende del funcionamiento de la biosfera.

- Un sistema que produce residuos que mediante reciclaje pueden volver a ser parcialmente utilizaos.

- Por tanto hay que estudiar la capacidad de la biosfera para absorber estos desechos.

- El mantenimiento de la biodiversidad.

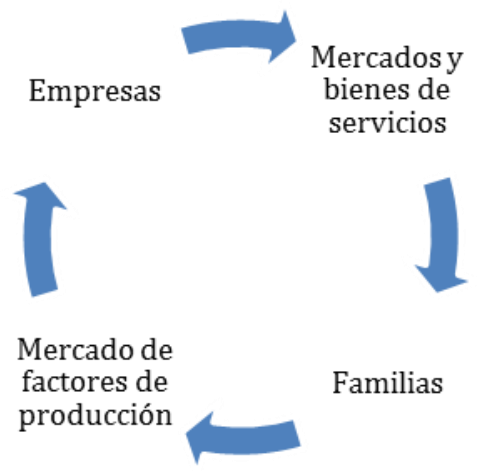

Figura 3. Proceso clásico de la economía 
En la figura 3 se muestra que en el proceso clásico de la economía solo intervienen dos factores, las familias y las empresas, donde las familias demandan los bienes y servicios mientras que las empresas asimilan y lo convierten en productos sin medir el impacto que esto produce a la economía. Lo ideal sería que las empresas evalúen si lo demandado por el mercado puede afectar directamente al ecosistema y luego emitir el criterio de producirlo, sin ser el beneficio económico el que esté por encima de la naturaleza.

El esquema a seguir sería el siguiente:

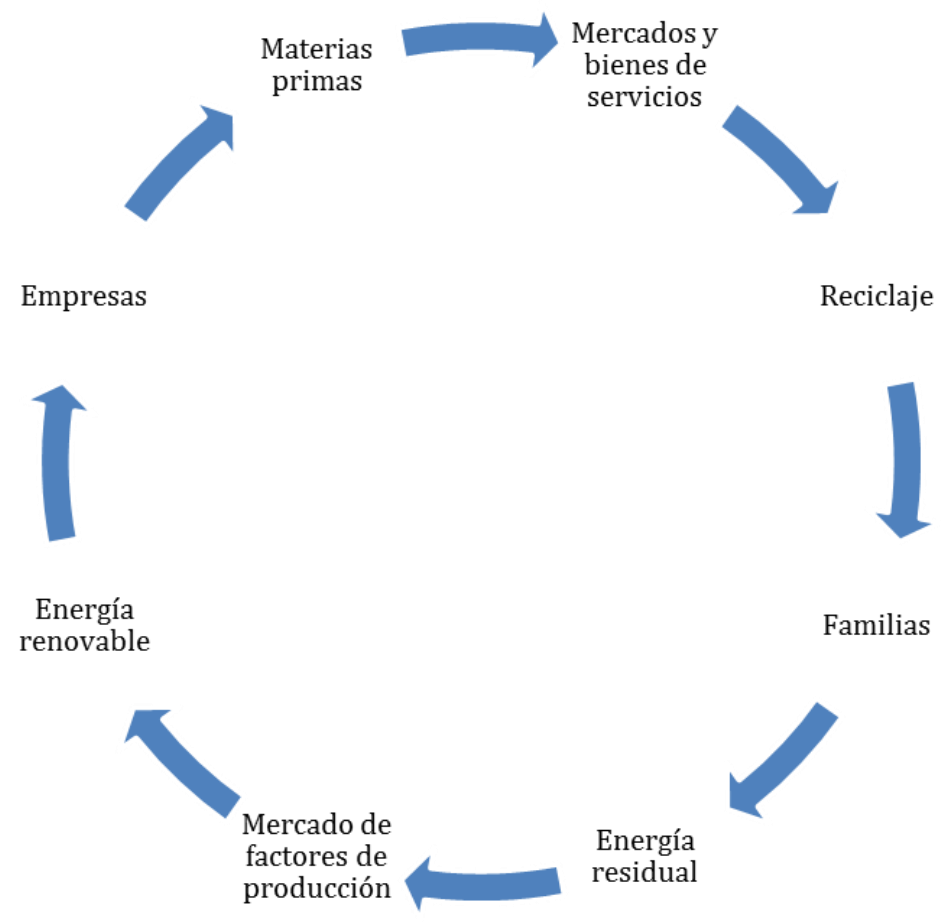

Figura 4. Proceso de la economía ecológica.

En la figura 4 se muestra que el esquema de la economía ecológica agrega que la demanda de bienes y servicios debe ser acompañado con el trabajo a base de la energía residual, es decir tomar aquellos productos inservibles para transformarlos en materias primas, luego estos pasan a la fábrica empleando en su proceso de producción energía solar como referencia de energía renovable.

Se finaliza este proceso con la puesta en marcha de un producto en el mercado con recomendaciones de ser reciclado para luego poder ser puesto en las manos del consumidor final, tratando que el mismo lo devuelva a la fábrica o empresa en forma de materia prima separada y dispuesta a ser transformado en un nuevo producto.

\section{Características de la economía ecológica.}

La economía ecológica entiende que la actividad económica no es una actividad que solo utilice bienes ambientales o recursos naturales de manera aislada, sino que está precisamente centrada en la utilización de los ecosistemas. Además investiga aspectos que quedan ocultos por 
un sistema de precios que infravalora la escasez y los perjuicios ambientales y sociales actuales y futuros.

Se articula sobre algunas nociones biofísicas fundamentales como la imposibilidad:

- De generar más residuos de los que puede tolerar la capacidad de asimilación de los ecosistemas.

- De extraer de los sistemas biológicos más de lo que puede ser considerado como su rendimiento sustentable o renovable.

- Por tanto se debe recomendar:

- El uso de los recursos renovables con un ritmo que no exceda su tasa de renovación.

- Usar los recursos agotables como el petróleo, el carbón, gas natural en un ritmo no superior al de su sustitución por recursos renovables.

- Solo generar residuos en la cantidad que el ecosistema sea capaz de asimilar o reciclar.

- Conservar la diversidad biológica.

- Quiere comprender los efectos sobre los ecosistemas para su adecuada gestión. Precisa, por tanto, crear nuevas instituciones y nuevas normas sociales de comportamiento. Se entiende que la escala de la economía está limitada por los ecosistemas y que gran parte del patrimonio natural no es substituible. Por ello los residuos solo pueden ser generados en una magnitud que el ecosistema pueda asimilar o sea capaz de reciclar.

Su objetivo central es la sostenibilidad ecológica de la economía, mientras que para la economía tradicional el crecimiento económico es su primera preocupación. Existe presencia de la economía cuando:

- Pone más énfasis en los riesgos tecnológicos que en las ventajas de las innovaciones, que estima que deben ser reflexionadas con base en el principio de precaución.

- Adopta una visión de largo plazo y que evalúa los costos y beneficios considerando los intereses del conjunto de la comunidad.

Precisa del desarrollo de indicadores biofísicos que permitan superar la insuficiencia de los indicadores, exclusivamente monetarios, para medir la sostenibilidad ecológica. Así, por ejemplo plantea el uso de los recursos renovables como la pesca en un ritmo que no exceda su tasa de renovación o el uso de los recursos no renovables, como el petróleo o la minería, en un ritmo no superior al necesario para su sustitución por recursos renovables.

Como se ha indicado, este es su punto más débil, hay que fijar de forma arbitraria unos límites ecológicos de la economía. Por tanto se está bajo una economía muy politizada y manipulable que, como todo en política, sobrepasa tanto la racionalidad económica como la racionalidad ecológica.

Enfoque económico desde el sector humano.

En función a la economía tradicional se tienen las siguientes premisas:

- $\quad$ Representa los productos del sector humano que van al sector humano. 
- Se abstrae de todas las relaciones con el resto de los casilleros de la tabla yua que considera como insumo primario al trabajo humano y no a los recursos naturales, y como producto final al consumo doméstico y no a los residuos o desperdicios que retornan al medio ambiente.

- Enfoque ambiental desde el sector no humano.

- Representa los productos del sector humano que van al sector no humano.

- Estudia la contaminación resultante de la inyección de los desperdicios de la economía en la naturaleza.

- Según esta visión, la contaminación no constituye un obstáculo fundamental al crecimiento; se trata, simplemente, de internalizar en los precios, los costos externos a través de impuestos o de la redefinición de derechos de la propiedad.

- Enfoque de la economía de los recursos naturales sector humano.

- Representa los insumos del sector no humano que van al sector humano.

- Estudia la extracción y agotamiento de los recursos naturales no renovables y el manejo de los recursos renovables, con una visión en que predomina la idea de que los recursos no constituyen realmente una limitación para el crecimiento económico.

- Enfoque Economía ecológica o ecología.

- Representa los productos del sector no humano que van al sector no humano.

- Los subsectores podrían clasificarse como plantas, animales, bacterias, hidrosfera, atmósfera, etc.

- Los ecólogos tradicionalmente han ignorado al sector humano.

\section{Metodología.}

Para relacionar el tema explicado sobre la procedencia de la economía ecológica es necesario tomar en cuenta ciertas prácticas que las empresas en la actualidad han formado parte de su modelo de negocios. Por lo tanto el tratamiento de la información está basado por un análisis de investigación cualitativa, ya que se describen solo las acciones producto de las causas de cuidar el medio ambiente, además del efecto económico que tiene en sus clientes o las empresas que se desarrollan en un determinado sector.

Para Hurtado (2014) indica que la investigación cualitativa acumula los criterios de diferentes especialistas sobre el tema para luego emitir una conclusión final para aportar con un conocimiento adicional al investigador. Para el presente tema se toman como ejemplo las acciones que han impulsado el gobierno, las empresas privadas y recomendaciones para el público en general de cómo al consumir y al mismo tiempo aportar con el cuidado del planeta.

Como referencia se emplea el muestreo aleatorio simple, en donde se tomará como ejemplo un caso de cada sector para luego emitir la conclusión sobre el tema. Algunos grupos activistas en generación al medio ambiente han sugerido que se debe crear energía renovable aprovechando la fuerza de la naturaleza como punto de partida para no parar la economía.

Presentación de resultados.

A continuación se muestran ejemplos sobre economía ecológica previa a la discusión.

Ejemplos de economía ecológica a nivel estatal. 
Partiendo desde el punto de vista teórico, "los impuestos son pagos forzosos desde el sector privado hacia el Estado, por los cuales no existe una contraprestación por parte de este último" (Musgrave, 1991).

Es decir que las personas están obligadas a cancelar un valor sobre las actividades que realizan a favor del Estado, a cambio el Estado funciona regulando la actividad del comercio y cubriendo servicios básicos además de proporcionar igualdad entre los habitantes del país. Tomando como referencia este criterio, los impuestos ambientales o denominados impuestos verdes son aquellos valores adicionales que se deben pagar sobre bienes o servicios considerados como contaminantes.

Para Olivia (2011) el uso de impuestos ambientales pueden generar algunas ventajas en comparación al objetivo de control sobre una actividad: produce eficiencia estática, de manera indirecta los impuestos ambientales reduzcan niveles de contaminación, volviendo más caros estos productos de manera que el consumidor los deje de comprar si su perspectiva en el precio no es la necesidad de cubrir un producto más caro.

Genera un beneficio de eficiencia dinámica, esto es en función hacia el elemento anterior ya que busca que los fabricantes del producto contaminante innoven sobre alternativas de producción más limpias y menos costosas, retribuyendo en algunos casos con la exoneración de impuestos como ejemplo para que lo sigan otras empresas.

En el año 2012 el Servicio de Rentas Internas incluyó en la matrícula un pago de impuestos a los vehículos donde se pagaba un valor sobre el avalúo del vehículo por concepto de contaminación mientras que los vehículos híbridos tenían que grabar un ICE sobre el valor de su automóvil, esto se muestra en la siguiente tabla.

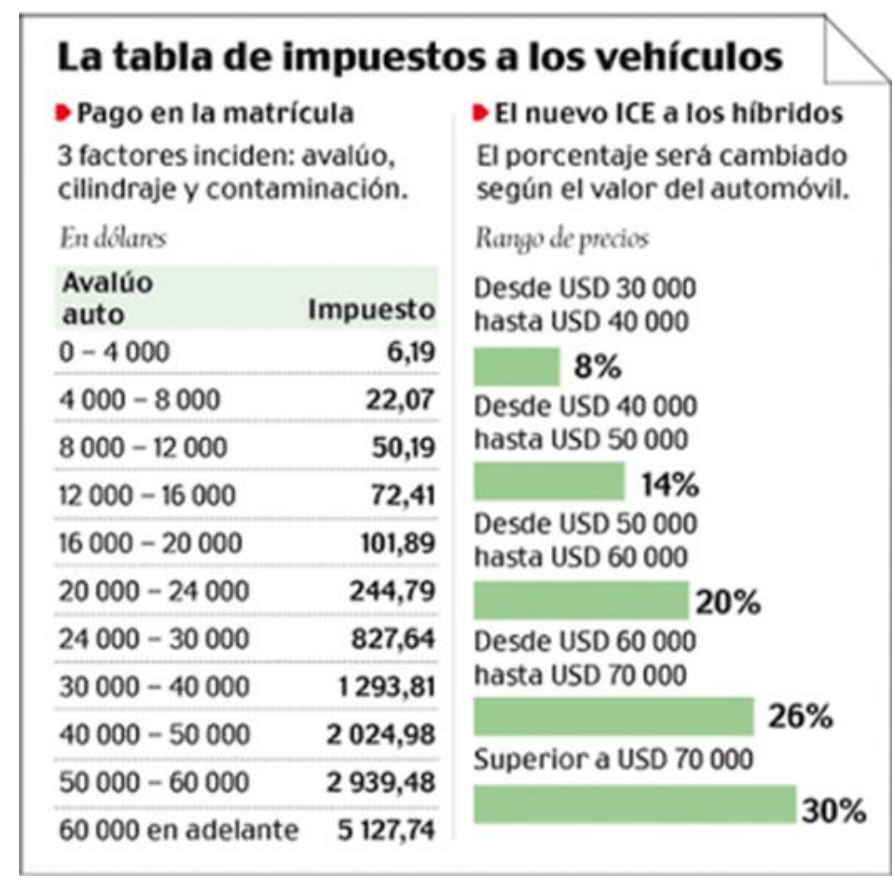

Figura 5. Tabla de impuestos a los vehículos. 
También se estableció un impuesto de dos centavos a las botellas no retornables a finales del año 2011, para que las personas dejen de consumir este tipo de material, pero si las reciclan recuperan estos dos centavos en la recicladora u embotelladoras, pero para el consumidor esto representa un ingreso mínimo que les cuesta más el pasaje de transporte y el tiempo que el valor recuperado.

\section{Ejemplos de economía ecológica a nivel privado.}

Un ejemplo que incentivó la compañía Diners es la recolección de libros que sean utilizados en escuelas de bajos recursos, de manera que los mismos no tengan que solicitar versiones actualizadas incentivando a la tala de árboles en el territorio para abastecer sus bibliotecas, esta campaña se la describe a continuación.

La campaña Dona un Buen Libro comenzó en el año 2008 y actualmente en vigencia, busca principalmente para resolver la problemática de proporcionar libros a los niños de escuelas de bajos recursos, a fin de instalar una biblioteca, que sirva de apoyo en temas de investigación y aprendizaje. Como antecedente se tiene que siete de cada diez niños en el Ecuador no tienen acceso a una biblioteca ni familiaridad con los libros.

Para esta campaña se obtuvo el apoyo de entidades públicas como el Ministerio de Educación, la Red del Buen Gobierno y el Consejo provincial del Pichincha. También se contó con el apoyo de tres principales centros comerciales de la ciudad de Quito, donde se ubicaron los contenedores de libros. El aporte de Diners en el Ecuador para la iniciativa Dona un Libro, se centró en el diseño y coordinación de esta propuesta y en la elaboración de una estrategia de comunicación.

Para la clasificación y selección de los libros, la Red de Buen Gobierno apoyo con un equipo técnico. La cantidad de libros recaudados fue de 115.377 libros, los cuales fueron donados por parte de Colegios, Parroquias, Centros Comerciales, Universidades, Editoriales, empresas y Personas particulares.

Otro ejemplo de economía ecología fue en el año 2013 donde la telefónica Movistar aprovecho sus centros de atención al cliente para reciclar cerca de 112 mil teléfonos celulares con el fin de concientizar el cuidado del medio ambiente y el tratamiento adecuado del reciclaje de equipos obsoletos, premiando a sus clientes con un saldo promocional de \$ 15 dólares consumibles de movistar a movistar.

La campaña publicitaria que implementó Telefónica ascendió a 2 millones de dólares, de esta manera la empresa demostró que por encima del beneficio económico de comercializar celulares, existe el compromiso que los componentes de equipos en desuso sean reciclados, empleando componentes para nuevos equipos o desechando de manera adecuada circuitos que son imposibles usarlos.

\section{Discusión}


La aplicación de una economía ecológica puede estar enlazada al modelo de negocios o ser creada a través de programas que promuevan el cuidado del medio ambiente. Según el concepto tradicional de la economía, el desarrollo social está basado en el consumo y la explotación, sin embargo se olvida del concepto del impacto económico, mientras que la ecología mide su relación de la naturaleza con el ser humano, sin hacer referencia a su explotación sino convivencia en armonía.

Desde el punto de vista administrativo esto no asegura el manejo eficiente de los recursos ya que depende si la producción o los procesos afectan o no al medio ambiente. Por lo tanto debe haber un punto de equilibrio donde la inversión obtenida sea recuperada y que los accionistas o dueños de empresa sacrifiquen un poco de beneficio por emplear energía más limpia y además competitivos para no modificar el precio del consumidor haciéndolos menos competitivos.

Finalmente en el Ecuador por parte del Gobierno Nacional a través de impuestos verdes y empresas privadas con programas en función al reciclaje y al uso de productos que se pensarían obsoletos, se ha logrado que se demanden más medios de producción y generar contaminación al planeta. Esta alternativa trata de motivar a las empresas a innovar sus procesos, elevar la calidad del producto y a evitar priorizar el beneficio y la salida del producto de manera más rápida sin medir el impacto ambiental en el planeta.

\section{Conclusiones}

La economía tradicional que impulsó la revolución industrial busca encontrar procesos automatizados, empleados por materiales que no tienen una previa evaluación de efectos contaminantes en el planeta, lo que incentiva a que las empresas generen gases de efecto invernadero que no solo afectan su normal giro del negocio sino que tienen efectos en toda la comunidad.

Para buscar un comparativo en la aplicación de la economía ecología se empleó datos bibliográficos sobre los acontecimientos en el Ecuador a nivel estatal y privado, tomando un ejemplo de cada uno de ellos de cómo en el país se aplica el concepto de economía ecológica en este tipo de control sin que esto lleve a la quiebra a las empresas, pero concientizando a las personas sobre las maneras en cómo cuidar al planeta.

En el año 2011 se empezó una política gubernamental a través de impuestos verdes en las botellas que incremento el precio sobre las bebidas, además de un valor adicional por contaminación en la matrícula anual de los vehículos, como una manera de desincentivar el consumo contaminante y retribuir un poco al planeta con menos cantidades de CO2.

\section{Bibliografía}

Banco Mundial. (2016). Emisiones de CO2. Obtenido de http://datos.bancomundial.org/indicador/EN.ATM.CO2E.PC?end=2013\&locations=CN\& start $=1960 \&$ view $=$ chart 
Barcia, W. (5 de Junio de 2012). Educándonos en el Ámbito Económico. Obtenido de http://ambitoeconomico.blogspot.com/2012/06/desarrollo-sostenible-de-la-poblaciony.html

Barquero, A. (2011). Administración de recursos humanos. San José: Universidad Estatal a Distancia.

Boland, L. (2011). Funciones de la administración. Buenos Aires: Red de Editoriales Universitarias Unidas.

Cárdenas, M. (2009). Naturaleza y propósito de la organización. El Cid Editor.

Cely, G. (2011). Temas de bioética ambiental. Bogotá: Javeriano.

Chaves, E. (2013). Administración de materiales. Barcelona: Pearson.

Dornbusch, R. y. (1996). Macroeconomía. Berlin: MC Grawhill.

Emery, D. (2011). Fundamentos de Administración Financiera. Atlanta: Pearson.

Fernández, R. (2013). La dimensión económica del Desarrollo Sostenible. San Vicente: Editorial Club Universitario.

Fred, D. (2013). Administración estratégica. México: Pearson Education.

Gómez, J. (2012). Economía y valores humanos. Madrid: Ediciones Encuentro.

Gómez, M. (2011). Introducción a la metodología de la investigación científica. Córdoba: Editorial Brujas.

Heizer, J. (2014). Principios de administración de operaciones. México: PEARSON Educación.

Hitt, M. (2002). Administración. Madrid: Pearson.

Hurtado, J. (2014). Metodología de la investigación holística. Caracas: Fundación Sypal 1era. Edición.

Krugman, P. (2008). Fundamentos de economía. Barcelona: Editorial Reverte S.A.

Lawrence, G. (2012). Principios de administración financiera. México D.F.: Pearson Education.

Levin, R. I. (2013). Estadística para Administración y Economía. Barcelona: Person.

Moguel, E. A. (2013). Metodología de la Investigación. México: Colección Héctor Merino Rodríguez. 
Musgrave, R. (1991). Hacienda Pública Teórica y Aplicada. Madrid: McGraw-Hill/Interamericana de España S.A.

Navarro, D. (2012). Administración financiera. Manizales: Universidad Nacional de Colombia.

Olivia, N. (2011). Impuestos verdes ¿una alternativa viable para el Ecuador? Quito: Servicio de Rentas Internas.

Owen, R. (2011). Administración Moderna. (G. Noriega, Ed.) Mexico: Limusa S.A.

Reyes, A. (2012). Administración de empresas: Teoría y Práctica. México: LIMUSA.

Robbins, S. (2005). Administración. Nueva York: Pearson Educación.

Robbins, S. P. (2013). Comportamiento Organizacional. México: Pearson Educación.

Summers, D. (2014). Administración de la calidad. México: Pearson Educación.

Tagiuri, R., \& Litwin, G. (1968). Organizational climate. Harvard University.

Vadillo, S. (2005). Administración de remuneraciones. México: Limusa S.A. 